\title{
Walking near a conformal fixed point
}

\author{
P. de Forcrand \\ Institute for Theoretical Physics, ETH Zürich, CH-8093 Zürich, Switzerland \\ and \\ CERN, Physics Department, TH Unit, CH-1211 Genève 23, Switzerland \\ E-mail: forcrandephys.ethz.ch
}

\section{Pepe*}

INFN, Istituto Nazionale di Fisica Nucleare, Sezione di Milano-Bicocca

Edificio U2, Piazza della Scienza 3, 20126 Milano, Italy

E-mail: pepe@mib.infn.it

\section{U.-J. Wiese}

Albert Einstein Center for Fundamental Physics, Institute for Theoretical Physics, Bern University, Sidlerstrasse 5, CH-3012 Bern, Switzerland

E-mail: wieseditp.unibe.ch

\begin{abstract}
We investigate the 2-d $O(3)$ model with a $\theta$-term as a toy model for slowly walking 4-d nonAbelian gauge theories. Using the very efficient meron-cluster algorithm, an accurate investigation of the scale dependence of the renormalized coupling is carried out for different values of the vacuum angle $\theta$. Approaching $\theta=\pi$, the infrared dynamics of the 2-d $O(3)$ model is determined by a non-trivial conformal fixed point. We provide evidence for a slowly walking behavior near the fixed point and we perform a finite-size scaling analysis of the mass gap.
\end{abstract}

The 30 International Symposium on Lattice Field Theory - Lattice 2012,

June 24-29, 2012

Cairns, Australia

${ }^{*}$ Speaker. 


\section{Introduction}

The Higgs mechanism is responsible for electroweak symmetry breaking in the Standard Model of particle physics. It is based on introducing a scalar field - the Higgs field - that gives mass to the weak bosons $W^{ \pm}$and $Z^{0}$ and to the fermions. Although this mechanism provides a very successful description of the electromagnetic and weak interactions, there are theoretical arguments which suggest that it is a low-energy description of some other phenomenon. In fact, because of radiative corrections, an unnatural fine-tuning to enormous precision has to be performed. Moreover, in the context of a grand unified approach, there is a hierarchy of very different energy scales that cannot be explained in a natural way. Some extensions of the Standard Model propose that the Higgs field is an effective field which results from a fermion-antifermion condensate. Other important phenomena in Nature show a similar behavior. For instance, superconductivity can be described by an Abelian-Higgs model where the Higgs field is an effective field following from the condensation of Cooper pairs. Another example is given by QCD: it has a low-energy effective description given by Chiral Perturbation Theory and based on the $\bar{q} q$ quark condensate.

Technicolor models rely on a similar approach and date back to the late ' 70 s $[1,2,3]$. The idea is to replace the Higgs sector of the Standard Model by a high energy variant of QCD, involving technigluons and techniquarks undergoing spontaneous chiral symmetry breaking and producing a condensate. This approach is successful from the general viewpoint but has problems when it is confronted with accurate electroweak precision measurements [4]. In particular, there are problems in suppressing contributions to flavor changing neutral currents and obtaining heavy fermion masses. One way out of this problem has been to endow a technicolor-extended Standard Model [5] with an infrared dynamics that differs from QCD. In QCD, the $\beta$-function has a single ultraviolet fixed point and the renormalized coupling increases quickly as one moves towards low energy scales. A different infrared dynamics takes place when the $\beta$-function has a second fixed point. In this case, the renormalized coupling no longer increases indefinitely as one considers low energy scales but it approaches a constant value determined by the infrared fixed point [6]. The actual position of the infrared fixed point depends on the renormalization scheme. The existence of a second fixed point is a feature of the specific theory, namely it is determined by the gauge symmetry group, by the number of fermionic flavors, and by their representations. However, it is important to keep in mind that the existence of a non-trivial fixed point can eventually depend on the scheme [7].

Yet another infrared dynamics happens when the $\beta$-function almost touches zero and thus gets close to having a second fixed point. In this situation, the renormalized coupling quickly increases as the energy scale is lowered from the ultraviolet. However, when the $\beta$-function is almost vanishing, the renormalized coupling becomes practically independent of the energy scale. Hence the renormalized coupling does not run but it "walks" and the theory is said to show a slowly walking behavior $[8,9,10]$. Only when one reaches very low energy scales, the $\beta$-function is no longer small and the renormalized coupling starts to increase again.

It has been shown that slowly walking technicolor models may solve the difficulties to describe the experimental data and they turn out to be possible candidates for the mechanism of electroweak symmetry breaking. Many investigations have been carried out to study whether there are nonAbelian gauge theories with some fermionic matter content showing a slowly walking behavior. In order to address this problem from first principles, one must perform non-perturbative numerical 
investigations using the lattice formulation; see, for instance, $[11,12,13,14,15,16,17,18]$. This is very challenging from the numerical viewpoint and it often happens that the results collected by different groups using different approaches are not consistent with each other. Hence it is very helpful to have an asymptotically free simple model which displays walking behavior that can be studied with very high accuracy and that can be used as a benchmark for this kind of investigations. A model with these properties is the 2-d $O(3)$ model with a $\theta$-term $[19,20]$.

\section{The 2-d $\mathrm{O}(3)$ model with a $\theta$-term}

The 2-d $O(3)$ model shares many important features with 4-d non-Abelian gauge theories. It is asymptotically free and it has a non-perturbatively generated mass gap. The Euclidean action in the continuum is given by

$$
S[\vec{e}]=\frac{1}{2 g^{2}} \int d^{2} x \partial_{\mu} \vec{e} \cdot \partial_{\mu} \vec{e},
$$

where $\vec{e}(x)$ is a 3-component unit vector defined at the point $x$ of a 2-d space-time and $g$ is the coupling constant. The model has instantons and topological sectors characterized by a topological charge $Q$ defined by

$$
Q[\vec{e}]=\frac{1}{8 \pi} \int d^{2} x \varepsilon_{\mu v} \vec{e} \cdot\left(\partial_{\mu} \vec{e} \times \partial_{v} \vec{e}\right) \in \Pi_{2}\left[S^{2}\right]=\mathbb{Z} .
$$

Similar to non-Abelian gauge theories, this leads to non-trivial $\theta$-vacuum effects when a term $i \theta Q[\vec{e}]$ is added to the action.

The topological susceptibility of the 2-d $O(3)$ model is logarithmically divergent in the ultraviolet cutoff but the 2-point function of the topological charge density is a well-defined quantity. This poses the question whether the concept of topological sectors is meaningful or not and whether $\theta$ is a relevant parameter or it just renormalizes to zero non-pertubatively. It turns out that $\theta$ is a relevant parameter which distinguishes different quantum field theories [21].

The 2-d $O(3)$ model is integrable at $\theta=0[22,23,24]$ and $\theta=\pi[25]$ but not at intermediate values. The mass gap $M$ at $\theta=0$ is given by $M=\frac{8}{e} \Lambda_{\overline{M S}}$ in the $\overline{M S}$ scheme [26]. Since perturbation theory is blind to the vacuum angle $\theta$, the same energy scale $\Lambda_{\overline{M S}}$ is present in the spectrum for every value of $\theta$. This property is valid also non-perturbatively. The mass gap for the $O(3)$ model has been computed analytically at $\theta=0$ [33] and $\theta=\pi$ [34] also in a finite spatial volume $L$.

Based on the exact S-matrix [25], the 2-d $O(3)$ model at $\theta=\pi$ at energies much lower than $\Lambda_{\overline{M S}}$ has an effective description given by the $k=1$ Wess-Zumino-Novikov-Witten (WZNW) model $[27,28,29]$ which has the following Euclidean action

$$
S[U]=\frac{1}{2 g^{\prime 2}} \int d^{2} x \operatorname{Tr}\left[\partial_{\mu} U^{\dagger} \partial_{\mu} U\right]-2 \pi i k S_{W Z N W}[U] .
$$

The matrix-valued field $U(x)$ belongs to $S U(2)=S^{3}$ and $g^{\prime}$ is a coupling constant. The WZNW term, $S_{W Z N W}$, is given by

$$
S_{W Z N W}[U]=\frac{1}{24 \pi^{2}} \int_{H^{3}} d^{2} x d x_{3} \varepsilon_{\mu v \rho} \operatorname{Tr}\left[U^{\dagger} \partial_{\mu} U U^{\dagger} \partial_{v} U U^{\dagger} \partial_{\rho} U\right]
$$


where $H^{3}$ is a 3-d hemisphere whose boundary $\partial H^{3}=S^{2}$ is the compactified 2-d space-time. The WZNW model has a global chiral symmetry $S U(2)_{L} \times S U(2)_{R} \sim O(4): U(x)^{\prime}=L U(x) R^{\dagger}$. Hence, at low energy, the $O(3)$ symmetry is enlarged to $O(4)$. The interesting feature here is that the $k=1$ WZNW model is a conformal field theory which makes the 2 -d $O(3)$ model at $\theta \approx \pi$ an ideal theoretical laboratory to investigate a slowly walking asymptotically free theory near an infrared conformal fixed point.

\section{The numerical study}

We regularize the 2-d $O(3)$ model on a triangular lattice. The lattice action is the standard nearest-neighbor action with the additional constraint than the angle between neighboring spins cannot be larger than $2 \pi / 3$ :

$$
S[\vec{e}]=\sum_{\langle x y\rangle} s\left(\vec{e}_{x}, \vec{e}_{y}\right), \quad s\left(\vec{e}_{x}, \vec{e}_{y}\right)=\frac{1}{g^{2}}\left(1-\vec{e}_{x} \cdot \vec{e}_{y}\right),
$$

for $\vec{e}_{x} \cdot \vec{e}_{y}>-\frac{1}{2}$ and $s\left(\vec{e}_{x}, \vec{e}_{y}\right)=\infty$ otherwise. We then add a $\theta$-term involving the topological charge $Q$. We use the geometric definition of the topological charge [30], given by the sum of the charge densities associated with each individual triangle. The charge density $q_{\langle x y z\rangle} \in\left[-\frac{1}{2}, \frac{1}{2}\right]$ of a triangle $\langle x y z\rangle$ is given by the area of the spherical triangle defined by the spins at the corners $x, y$, and $z$

$$
R \exp \left(2 \pi i q_{\langle x y z\rangle}\right)=1+\vec{e}_{x} \cdot \vec{e}_{y}+\vec{e}_{y} \cdot \vec{e}_{z}+\vec{e}_{z} \cdot \vec{e}_{x}+i \vec{e}_{x} \cdot\left(\vec{e}_{y} \times \vec{e}_{z}\right), \quad R \geq 0 .
$$

The partition function is then given by

$$
Z(\theta)=\prod_{x} \int_{S^{2}} d \vec{e}_{x} \exp (-S[\vec{e}]+i \theta Q[\vec{e}])
$$

The aim of this study is to investigate the scale dependence of a renormalized coupling and observe the change from running to walking as we approach $\theta=\pi$. We consider the mass gap scheme [31, 32] and we define the running coupling constant $\alpha(\theta, L)=g^{2}(\theta, L) \equiv m(\theta, L) L$ through the mass gap $m(\theta, L)$ in a periodic volume of spatial size $L$. The corresponding $\beta$-function is given by $\beta(\theta, \alpha)=-L \partial_{L} \alpha(\theta, L)$. The mass gap is obtained from the zero-momentum two-point function

$$
C\left(t_{1}, t_{2} ; \theta\right)=\frac{1}{Z(\theta)} \prod_{x} \int_{S^{2}} d \vec{e}_{x} \vec{E}\left(t_{1}\right) \cdot \vec{E}\left(t_{2}\right) \exp (-S[\vec{e}]+i \theta Q[\vec{e}]) \sim \exp \left(-m(\theta, L)\left(t_{2}-t_{1}\right)\right),
$$

where $\vec{E}(t)=\sum_{x_{1}} \vec{e}_{x}$ is the average of the spins belonging to a given time-slice $x=\left(x_{1}, t\right)$. The mass gap at finite volume can be computed analytically both at $\theta=0$ [33] and at $\theta=\pi$ [34]. Since the infinite volume mass gap $M$ at $\theta=0$ does not vanish, the renormalized coupling $\alpha(0, L) \rightarrow M L$ increases linearly for large $L$. Instead, at $\theta=\pi$, the mass gap vanishes and the coupling approaches the fixed point $\alpha(\pi, L) \rightarrow \alpha^{\star}=\pi$ as $L \rightarrow \infty$. Thus, the $\beta$-function has a second fixed point at $\alpha^{\star}=\pi$, i.e. $\beta(\theta=\pi, \alpha=\pi)=0$ [37], in addition to the Gaussian fixed point at $\alpha=0$. The fixed point of the $\beta$-function at $\theta=\pi$ is a double zero and expanding near this fixed point shows that large logarithmic finite-size corrections are present:

$$
\beta(\alpha) \approx-C\left(\alpha-\alpha^{\star}\right)^{2} \Rightarrow \alpha(L) \approx \alpha^{\star}-\frac{1}{C \log \left(L / L_{0}\right)} .
$$



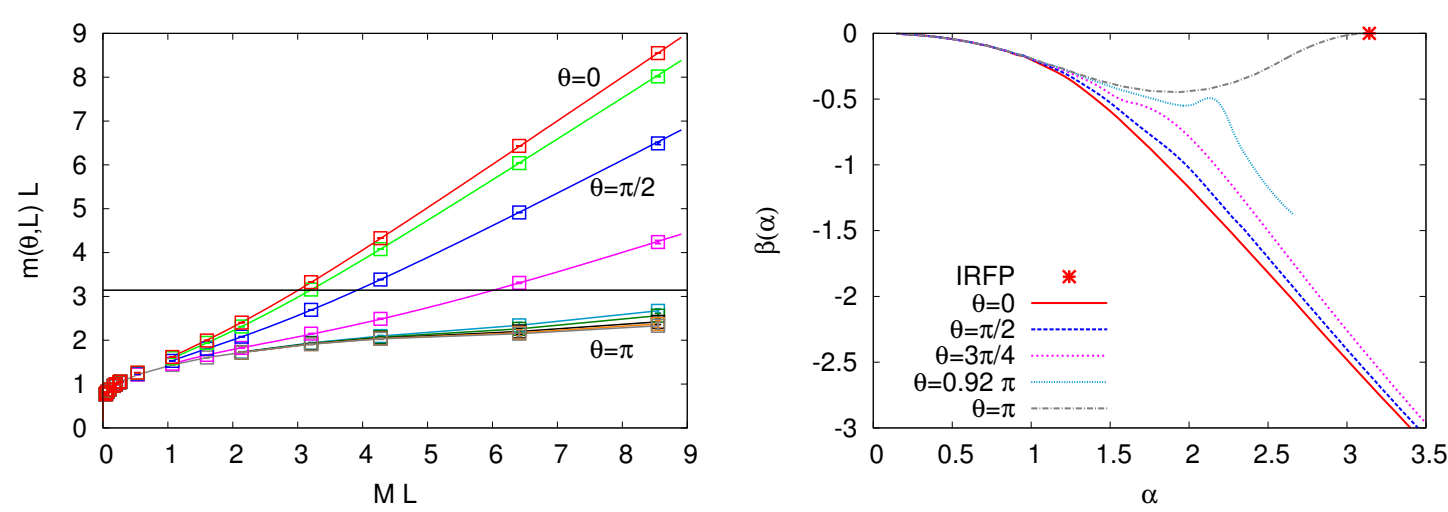

Figure 1: (Left) The running coupling constant $\alpha(\theta, L)=m(\theta, L) L$ as a function of the scale set by the spatial size $L$ for $\theta / \pi=0,0.25,0.50,0.75,0.92,0.94,0.96,0.98$ and 1 . The horizontal line is the fixed point value $\pi$. (Right) The corresponding $\beta$-function obtained by differentiation of the numerical data; the exact values at $\theta=\pi$ are from [34].

These logarithmic corrections are responsible for a very slow approach to the conformal fixed point and they are a general feature of a walking theory. In the case of the 2-d $O(3)$ model with a $\theta$-term, they are associated with a marginally irrelevant operator that breaks the $O(4)$ symmetry down to $O(3)[35,36,37,38,39]$.

The numerical simulation of the 2-d $O(3)$ model with a $\theta$-term is non-trivial: the contribution of the $\theta$-term to the Boltzmann factor is oscillatory and leads to a severe sign problem. However, in this case, the meron-cluster algorithm [40] partially tames the problem, making the numerical simulations feasible. We have performed numerical simulations choosing the renormalization point $\alpha\left(\theta=0, M r_{0}=0.2671536\right)=m\left(\theta=0, M r_{0}=0.2671536\right) r_{0}=1.0595[32,41]$. Then we have investigated the behavior of the renormalized coupling at the scales $M L=s M r_{0}$ with $s=0.15, \ldots, 1$ in the ultraviolet region and with $s=2, \ldots, 32$ in the infrared regime. The data from numerical simulations have been complemented with 3-loop analytic perturbative results in the range $s=10^{-16}, \ldots, 0.1$ [32]. At each energy scale $s$ we first take the continuum limit considering 4-5 different values for the lattice spacing.

The left panel of figure 1 shows the dependence of the renormalized coupling $\alpha(\theta, L)$ on the scale $L$ at various values of $\theta$. Except for $\theta \approx \pi$, the coupling clearly shows a running behavior and it ultimately rises linearly with a slope that decreases as $\theta \rightarrow \pi$. The curves for $0 \leq \theta \leq 3 \pi / 4$ show the effect of leading finite-size corrections $\exp (-m L) / \sqrt{m L}$ to the infinite-size mass gap $m$. The behavior changes close to $\theta=\pi$ and the running of the renormalized coupling slows down. At $\theta=\pi$ we know that the approach to the fixed point value $\pi$ is extremely slow with a $1 / \log (M L)$ correction. By spline interpolation, we derive the $\beta$-function that is shown in the right panel of figure 1. We observe the familiar running of the $\beta$-function for $\theta=0, \frac{\pi}{2}$. A different behavior starts to show up for $\theta=\frac{3 \pi}{4}$ and it becomes evident for $\theta=0.92 \pi$. At $\theta=\pi$ we observe the conformal fixed point responsible for the walking behavior.

The infrared conformal fixed point at $\theta=\pi$ is a critical point and so it is interesting to perform a finite size scaling investigation of the mass gap. The dependence of the infinite-volume mass gap 
on $\theta$ is known [37]

$$
m(\theta, L \rightarrow \infty) \sim|\theta-\pi|^{2 / 3}|\log (|\theta-\pi|)|^{-1 / 2} .
$$

A direct observation of the collapse of the mass gap $m(\theta, L) L$ for various values of $L$ and $\theta$ on a single universal curve is practically impossible due to the very slow approach to the critical point. A better observable, insensitive to the large logarithmic corrections, is $m(\theta, L) L+[\pi-m(\pi, L) L]$. Figure 3 shows its behavior as a function of the finite size scaling variable $M L t^{2 / 3} / \sqrt{\left|\log \left(t / t_{0}\right)\right|}$, where $t$ is the reduced coupling $t=1-\theta / \pi$, and $t_{0}=70$. The data fall on a universal curve, confirming the critical exponent $2 / 3$ of the WZNW model in eq.(3.6). The curve displays both the slow walking near $\pi$, and the linearly rising regime as $L \rightarrow \infty$.

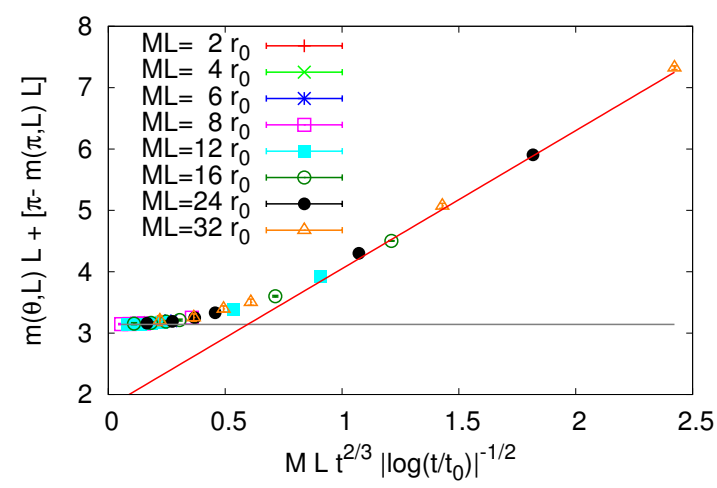

Figure 2: Finite-size scaling of $m(\theta, L) L+[\pi-m(\pi, L) L]$ near $\theta=\pi$ as a function of $M L t^{2 / 3} / \sqrt{\left|\log \left(t / t_{0}\right)\right|}$, with $t=1-\theta / \pi, t_{0}=70$. The spatial sizes $L$ are multiples of the renormalization reference scale $r_{0}=$ 0.26715356 .

\section{Conclusions}

We have investigated the 2-d $O(3)$ model with a $\theta$-term. It is an asymptotically free theory with a slowly walking behavior near the infrared conformal fixed point at $\theta=\pi$. It is a very interesting toy model for slowly walking 4-d non-Abelian gauge theories whose non-perturbative investigation is highly challenging. The $2-\mathrm{d} O(3)$ model with a $\theta$-term captures the relevant features of a nonAbelian slowly walking theory and thus provides an ideal theoretical laboratory where all these properties can be studied accurately.

\section{References}

[1] S. Weinberg, Phys. Rev. D13 (1976) 974.

[2] L. Susskind, Phys. Rev. D20 (1979) 2619.

[3] E. Farhi and L. Susskind, Phys. Rev. D20 (1979) 3404.

[4] M. E. Peskin and T. Takeuchi, Phys. Rev. Lett. 65 (1990) 964.

[5] C. Hill and E. Simmons, Phys. Rep. 381 (2003) 235. 
[6] T. Banks and A. Zaks, Nucl. Phys. B196 (1982) 189.

[7] E. Bilgici et al., PoS LAT 2009, 063 (2009).

[8] B. Holdom, Phys. Rev. D24 (1981) 1441.

[9] T. Appelquist and L. C. R. Wijewardhana, Phys. Rev. D35 (1987) 774.

[10] D. D. Dietrich, F. Sannino, and K. Tuominen, Phys. Rev. D72 (2005) 055001.

[11] T. Appelquist, G. T. Fleming, and E. T. Neil, Phys. Rev. Lett. 100 (2008) 171607.

[12] A. Deuzeman, M. P. Lombardo, and E. Pallante, Phys. Lett. B670 (2008) 41.

[13] A. J. Hietanen, K. Rummukainen, and K. Tuominen, Phys. Rev. D80 (2009) 094504.

[14] Z. Fodor, K. Holland, J. Kuti, D. Nogradi, and C. Schröder, JHEP 0911 (2009) 103.

[15] L. Del Debbio, B. Lucini, A. Patella, C. Pica, and A. Rago, Phys. Rev. D82 (2010) 014510.

[16] T. DeGrand, Y. Shamir, and B. Svetitsky, Phys. Rev. D82 (2010) 054503.

[17] A. Hasenfratz, Phys. Rev. Lett. 108 (2012) 061601.

[18] P. de Forcrand, S. Kim and W. Unger, arXiv:1208.2148 [hep-lat].

[19] D. Nogradi, JHEP 1205 (2012) 089.

[20] P. de Forcrand, M. Pepe and U.-J. Wiese, to appear in Phys. Rev. D, arXiv:1204.4913.

[21] M. Bögli, M. Pepe, F. Niedermayer, and U.-J. Wiese, JHEP 1204 (2012) 117.

[22] A. B. Zamolodchikov and A. B. Zamolodchikov, Ann. Phys. 120 (1979) 253.

[23] A. Polyakov and P. B. Wiegmann, Phys. Lett. B131 (1983) 121.

[24] P. B. Wiegmann, Phys. Lett. B152 (1985) 209.

[25] A. B. Zamolodchikov and V. A. Fateev, Sov. Phys. JETP 63 (1986) 913.

[26] P. Hasenfratz, M. Maggiore, and F. Niedermayer, Phys. Lett. B245 (1990) 522.

[27] J. Wess and B. Zumino, Phys. Lett. B37 (1971) 95.

[28] S. P. Novikov, Usp. Mat. Nauk. 37N5 (1982) 3.

[29] E. Witten, Commun. Math. Phys. 92 (1984) 455.

[30] B. Berg and M. Lüscher, Nucl. Phys. B190 (1981) 412.

[31] M. Lüscher, Phys. Lett. B118 (1982) 391.

[32] M. Lüscher, P. Weisz, and U. Wolff, Nucl. Phys. B359 (1991) 221.

[33] J. Balog and A. Hegedus, J. Phys. A37 (2004) 1881; Nucl. Phys. B725 (2005) 531; Nucl. Phys. B829 (2010) 425 .

[34] J. Balog, private communication.

[35] J. Cardy, J. Phys. A19 (1986) L1093.

[36] I. Affleck and F. D. M. Haldane, Phys. Rev. B36 (1987) 5291.

[37] I. Affleck, D. Gepner, H. J. Schulz, and T. Ziman, J. Phys. A22 (1989) 511.

[38] N. Read and R. Shankar, Nucl. Phys. B336 (1990) 457.

[39] D. Controzzi and G. Mussardo, Phys. Rev. Lett. 92 (2004) 021601.

[40] W. Bietenholz, A. Pochinsky, and U.-J. Wiese, Phys. Rev. Lett. 75 (1995) 4524.

[41] J. Balog, F. Niedermayer, and P. Weisz, Nucl. Phys. B824 (2010) 563. 